\title{
Estimation of the FRF Through the Improved Local Bandwidth Selection in the Local Polynomial Method
}

Thummala, Prasanth; Schoukens, Johan

Published in:

I E E E Transactions on Instrumentation and Measurement

Link to article, DOI:

10.1109/TIM.2012.2196393

Publication date:

2012

Link back to DTU Orbit

Citation (APA):

Thummala, P., \& Schoukens, J. (2012). Estimation of the FRF Through the Improved Local Bandwidth Selection in the Local Polynomial Method. I E E E Transactions on Instrumentation and Measurement, 61(10), 2833-2843. https://doi.org/10.1109/TIM.2012.2196393

\section{General rights}

Copyright and moral rights for the publications made accessible in the public portal are retained by the authors and/or other copyright owners and it is a condition of accessing publications that users recognise and abide by the legal requirements associated with these rights.

- Users may download and print one copy of any publication from the public portal for the purpose of private study or research.

- You may not further distribute the material or use it for any profit-making activity or commercial gain

- You may freely distribute the URL identifying the publication in the public portal 


\title{
Estimation of the FRF Through the Improved Local Bandwidth Selection in the Local Polynomial Method
}

\author{
Prasanth Thummala, Student Member, IEEE, and Johan Schoukens, Fellow, IEEE
}

\begin{abstract}
This paper presents a nonparametric method to measure an improved frequency response function (FRF) of a linear dynamic system excited by a random input. Recently, the local polynomial method (LPM) has been proposed as a technique to reduce the leakage errors on FRF measurements. The noise sensitivity of the LPM was similar to that of the classical windowing methods, while the leakage rejection is improved from an $O\left(N^{-1}\right)$ to an $O\left(N^{-3}\right)$. This paper shows a methodology, to automatically tune the parameter of the LPM, viz., the local bandwidth that sets how many neighboring frequency lines are combined in a single-point estimate, to get lower noise sensitivity by increasing the smoothening of the original LPM, without any user interaction. The balance between noise reduction and bias error will be automatically retrieved.
\end{abstract}

Index Terms-Bias-variance tradeoff, leakage rejection, local bandwidth, noise rejection, system identification.

\section{INTRODUCTION}

$\mathbf{L}$ INEAR dynamic models are very useful to understand, control, and predict physical processes and to design new products [1]-[4]. System identification provides a mathematical model for a dynamic system starting from measured inputs and outputs. The nonparametric modeling will be of particular interest even if a parametric modeling is the final goal for many reasons. Nonparametric methods are noniterative and require no user interaction. By providing the user the FRF of the plant and the power spectrum of the disturbing noise, it is easy to get an idea about the complexity of the modeling problem [1], [5], [6]. This gives a flexibility to verify the quality of the measurements at a very early stage of the experiment, giving the user the opportunity to improve the experiments if necessary. The FRF is a very attractive tool for the model validation/selection purposes. There also is a possibility to check for the presence of nonlinear distortions and their analysis before starting a more demanding parametric identification process [4], [7].

Manuscript received September 25, 2011; revised March 19, 2012; accepted March 20, 2012. This work was supported in part by the Fund for Scientific Research (FWO-Vlaanderen), by the Flemish Government (Methusalem), and by the Belgian Federal Government (IUAP VI/4). The Associate Editor coordinating the review process for this paper was Dr. Jesús Ureña.

P. Thummala is with the Department of Electrical Engineering, Technical University of Denmark, 2800 Kongens Lyngby, Denmark (e-mail: pthu@elektro.dtu.dk).

J. Schoukens is with the Department of Fundamental Electricity and Instrumentation (ELEC), Vrije University Brussel, 1050 Brussels, Belgium (e-mail: Johan.Schoukens@vub.ac.be).

Color versions of one or more of the figures in this paper are available online at http://ieeexplore.ieee.org.

Digital Object Identifier 10.1109/TIM.2012.2196393
The major problem in estimating the nonparametric FRF and the noise covariance matrix, using arbitrary excitation, is the suppression of the system and noise leakage errors while at the same time, the noise sensitivity should be as low as possible. The leakage errors are introduced when transforming a finite number $N$ of the time-domain samples to the frequency domain via the discrete Fourier transform (DFT). Even without disturbing noise, the FRF measurements are corrupted by these errors due to the windowing effects: Only a finite record length can be measured and processed [14]. These problems can be avoided by measuring an integer number of periods of the steady-state response of the system to a periodic excitation. This requires that the system transient response decayed well below the noise level, which is done at the cost of lost measurement time since the transient information cannot be used. In many applications, the user wants to stick to random excitations, either for technical reasons or by tradition in a given application field. However, in random excitations, the steady state is never reached.

Until the 1980s, leakage errors on the FRF measurements were studied at the input and output signal levels, without considering the linear relation between the input and output [1], [8]. In a series of papers [9]-[13], the importance of this relation was recognized: In FRF measurements, the errors are due to unknown past inputs and missing future outputs. Both the effects are highly structured with smooth frequency characteristics; this key observation leads to a new analysis of the existing methods and to a better performing method to estimate the FRF and the power spectrum of the disturbing noise [14]-[16], called "the local polynomial method" (LPM).

Elimination of the system and noise leakage errors via timedomain windowing is handled by spectral analysis methods. In the FRF estimation, we study the linear relation between two signals, while the spectral estimation is focused on a single signal [17]. For that reason, spectral modeling methods like autoregressive, autoregressive moving average are not the first choice to measure the FRF. A major problem is that the stated methods do not give an estimate of the phases. To reduce the noise on the FRF estimates, the record of $N$ samples is divided into $M$ subrecords of length $N / M$, which decreases the frequency resolution from $f_{s} / N$ to $M f_{s} / N$, and the results are averaged over the $M$ subrecords. Hence, choosing $M$ is making a tradeoff between, on the one hand, the leakage elimination and the frequency resolution (the larger the $M$, the larger the leakage errors and the smaller the frequency resolution) and, on the other hand, the noise suppression (the variance of the estimates decreases by $M$ ) [1]. The LPM does not split the data into subrecords. The basic assumption in the LPM is that the 
system and noise transfer functions are smooth functions of the frequency, which can be locally approximated by a low-degree polynomial. The LPM has a maximal frequency resolution $f_{s} / N$ and suppresses much better the system and noise leakage errors while maintaining a useful noise averaging effect that is at least as good as that of the spectral analysis methods, when making the comparison for a same frequency resolution.

This paper extends the LPM, to automatically tune the parameters of the LPM, without any user interaction, to get an improved balance between leakage rejection, noise rejection, and bias errors. The local bandwidth $(n)$ and order of the local polynomial approximation $(R)$ are the important parameters to be tuned in the LPM; between these, the most important parameter to be tuned is the local bandwidth, which decides how many neighboring frequencies are combined to generate the local point estimate. Increasing the local bandwidth reduces the variance (better smoothening) but increases the bias (increased interpolation error) so the bias-variance tradeoff is made, to tune the local bandwidth. If the FRF data are used as the input of a parametric estimation step, the only valid choice is to keep the bias errors as small as possible by selecting the smallest local bandwidth.

In Section II, we briefly describe the LPM. We discuss balancing of variance-bias in Section III. Next, we discuss how to select the improved local bandwidth of the method without user interaction in Section IV and eventually illustrate the proposed method on simulations in Section V. We made comparison of the proposed method with the classical windowing method (Hanning window) in Section VI, followed by the conclusions in Section VII.

\section{LPM}

In this section, we first describe the system and measurement setup in Section II-A; next, we discuss briefly the basic idea in Section II-B, and we formulate the LPM as a linear least squares problem that is solved frequency per frequency. Finally, we discuss very briefly the FRF estimation using a Hanning window in Section II-C. We refer the reader to [14] for a detailed theoretical study of the stated LPM.

\section{A. System}

In this paper, a linear discrete-time single-input single-output system $G(q)$ excited with random noise is considered

$$
y(t)=G(q) u(t)
$$

with $q^{-1}$ being the backward shift operator $\left(q^{-1} x(t)=\right.$ $x(t-1))$. The exact input $u(t)$ is assumed to be known, while the output is disturbed with additive noise $v(t)$. The noise $v(t)$ is assumed to be filtered white noise

$$
v(t)=H(q) e(t) .
$$

For an infinitely long data record $t=-\infty, \ldots, N-1$, the input-output relation is

$$
y(t)=G(q) u(t)+H(q) e(t) .
$$

For a finite record length $t=0, \ldots, N-1$, as in practical applications, (1) has to be extended with the initial condition (transient) effects of the dynamic plant $\left(t_{G}(t)\right)$ and the noise system $\left(t_{H}(t)\right)[4]$

$$
y(t)=G(q) u(t)+H(q) e(t)+t_{G}(t)+t_{H}(t) .
$$

Using the DFT

$$
X(k)=\frac{1}{\sqrt{N}} \sum_{t=0}^{N-1} x(t) e^{-j 2 \pi k t / N}
$$

an exact frequency-domain formulation of (2) is obtained

$$
Y(k)=G\left(\Omega_{k}\right) U(k)+H\left(\Omega_{k}\right) E(k)+T_{G}\left(\Omega_{k}\right)+T_{H}\left(\Omega_{k}\right)
$$

where the index $k$ points to the frequency $k f_{s} / N$ with $f_{s}$ being the sampling frequency, and the generalized frequency $\Omega_{k}=e^{j 2 \pi k f_{s} / N}$. The contributions $U(k), E(k)$, and $Y(k)$ are an $O\left(N^{0}\right)$, and the generalized transient terms of the plant and noise dynamics, due to beginning and end effects $T_{G}\left(\Omega_{k}\right)$ and $T_{H}\left(\Omega_{k}\right)$, are an $O\left(N^{-(1 / 2)}\right)$. It is most important for the rest of this paper to understand that (3) is an exact relation [4], [21]-[23]. The notation $O\left(N^{-\alpha}\right)$, called "ordo," stands for a variable that goes to zero at least as fast as $N^{-\alpha}$.

The transfer functions $G\left(\Omega_{k}\right)$ and $H\left(\Omega_{k}\right)$ as well as the leakage contributions $T_{G}\left(\Omega_{k}\right)$ and $T_{H}\left(\Omega_{k}\right)$ are continuous function of the frequency, and they are described by rational forms in $\Omega$ and, hence, are smooth functions of the frequency.

The LPM makes an optimal use of the smooth behavior of $G$ and $T_{G}$ to significantly reduce the leakage errors. This results in superior properties compared with the classical windowing methods.

\section{B. LPM: Basic Idea and Brief Description}

In this section, we give a brief introduction to the LPM. A detailed description, together with a full analysis, is given in [14]-[16]. The basic idea of the LPM is very simple: The transfer function $G$ and the transient terms $T_{G}$ and $T_{H}$ are smooth functions of the frequency so that they can be approximated in a narrow frequency band, around a user-specified frequency $k$ by a complex polynomial. The complex polynomial parameters are estimated from the experimental data. Next, the FRF $G_{k}\left(\Omega_{k}\right)$ obtained from a local bandwidth, centered around frequency $k$, is retrieved from this local polynomial model as the measurement of the FRF at the frequency $\Omega_{k}$.

Consider the equivalent relation for the DFT spectra equation (3), and rewrite it as

$$
\begin{aligned}
Y(k) & =G_{k}\left(\Omega_{k}\right) U(k)+T_{G}\left(\Omega_{k}\right)+H\left(\Omega_{k}\right) E(k)+T_{H}\left(\Omega_{k}\right) \\
& =G_{k}\left(\Omega_{k}\right) U(k)+T\left(\Omega_{k}\right)+V(k)
\end{aligned}
$$

where the generalized transient term $T\left(\Omega_{k}\right)$ accounts for the leakage of the plant and the noise dynamics with $T\left(\Omega_{k}\right)=$ $T_{G}\left(\Omega_{k}\right)+T_{H}\left(\Omega_{k}\right)=O\left(N^{-(1 / 2)}\right)$ [4], [21]. The remaining noise term is $V(k)=H\left(\Omega_{k}\right) E(k)$. The terms $G_{k}\left(\Omega_{k}\right) U(k)$ and $V(k)$ are an $O\left(N^{0}\right)$.

Making use of the smoothness of $G_{k}\left(\Omega_{k}\right)$ and $T\left(\Omega_{k}\right)$, the following Taylor series representation holds for the frequency 
lines $k+r$, with $r=0, \pm 1, \ldots, \pm n$

$$
\begin{aligned}
G_{k+r}\left(\Omega_{k+r}\right)= & G_{k}\left(\Omega_{k}\right)+\sum_{s=1}^{R} g_{s}(k) r^{s}+O\left(\left(\frac{r}{N}\right)^{(R+1)}\right) \\
T\left(\Omega_{k+r}\right)= & T\left(\Omega_{k}\right)+\sum_{s=1}^{R} t_{s}(k) r^{s} \\
& +\left(N^{-\frac{1}{2}}\right) O\left(\left(\frac{r}{N}\right)^{(R+1)}\right)
\end{aligned}
$$

where the remainder term $O\left((r / N)^{(R+1)}\right)$ is the polynomial interpolation error. Putting all the parameters $G_{k}\left(\Omega_{k}\right)$ and $T\left(\Omega_{k}\right)$ and the parameters of the Taylor series $g_{s}$ and $t_{s}$ with $s=$ $1, \ldots, R$ in a column vector $\theta$ and their respective coefficients in a row vector $K(k, r)$ allows (4) to be rewritten (neglecting the remainders) as

$$
Y(k+r)=K(k, r) \theta+V(k) .
$$

Collecting (6) for $r=-n,-n+1, \ldots, 0, \ldots, n$ finally gives

$$
Y_{n}=K_{n} \Theta+V_{n}
$$

with $Y_{n}, V_{n}$, and $K_{n}$ being the values of $Y(k+r), V(k+r)$, and $K(k, r)$ stacked on top of one another. Observe that the matrix $K_{n}$ depends upon $U$. Solving this set of equations [(7)] in the unknown $\Theta$, in least squares sense, eventually provides the polynomial least squares estimate for the FRF $\hat{G}_{k}\left(\Omega_{k}\right)$ centered around frequency $k$ [14], [16]. In order to get a full rank matrix $K_{n}$, enough spectral lines should be combined: $2 n+$ $1>2(R+1)$. The smallest interpolation error is obtained for $n=R+1$.

The LPM can also be used to solve the spectral estimation of the disturbing noise problem [14], [15]. The residual of the least squares fit of (7) is

$$
\hat{V}_{n}=Y_{n}-K_{n} \Theta=Y_{n} P_{n}
$$

with $P_{n}=I_{2 n+1}-K_{n}\left(K_{n}^{H} K_{n}\right)^{-1} K_{n}^{H}$ being an idempotent projection matrix and

$$
\hat{V}_{n}=V_{n} P_{n} .
$$

An estimate of the noise covariance matrix $C_{V}=\operatorname{cov}(V(k))$ where $V(k)=H\left(\Omega_{k}\right) E(k)$ is

$$
\hat{C}_{V}(k)=\frac{1}{q} \hat{V}_{n} \hat{V}_{n}^{H}
$$

with $q=2 n+1-2(R+1)=2(n-R)-1$. The main focus in this paper is to make an improved bias-variance tradeoff on the estimated FRF $\hat{G}_{k}\left(\Omega_{k}\right)$.

\section{Classical FRF Estimation Approach: Hanning Window}

The leakage errors on the FRF measurements were also reduced by the windowing methods to some extent [1], [14], [15], [18]

$$
\hat{G}_{\text {Hann }}\left(\Omega_{k}\right)=G\left(\Omega_{k}\right)+G_{\text {leakHann }}+G_{\text {intHann }}
$$

where

$G_{\text {leakHann }}=O\left(N^{-5 / 2}\right)$; system leakage error (the leakage error is reduced from an $O\left(N^{-1 / 2}\right)$ to an $O\left(N^{-5 / 2}\right)$ by applying a Hanning window);

$G_{\text {intHann }}=O\left(N^{-1}\right)$; interpolation error, which dominates the results.

The disadvantages of this approach are loss in the frequency resolution [1] and increased leakage errors as explained later in Section VI.

\section{BALANCING OF VARIANCE AND BIAS}

The LPM rejects leakage errors quite well when measuring the FRF. The errors of the LPM are studied in detail in [14] and [15]. In this paper, we propose a method to reduce also the noise sensitivity without any user interaction. Increasing the local bandwidth $n$ of the LPM will reduce the variance on the estimated FRF but increases the bias on it. We want to retrieve automatically the balance between the bias error and the noise rejection (variance error). The goal of this paper is to tune the choice for the local bandwidth $n$, without any user interaction for a fixed polynomial order $R$, while the system is solved with different local bandwidths. In Section III-A, we discuss the bias-variance tradeoff; next, we discuss improved balance of the bias and the variance in Section III-B.

\section{A. Bias-Variance Tradeoff}

The detailed derivations for the bias and the variance are given in [14]; in this paper, we discuss briefly about them.

The bias on the estimated FRF $\hat{G}_{k}\left(\Omega_{k}\right)$ is

$$
E\left\{\hat{G}_{k}\left(\Omega_{k}\right)\right\}=G_{k}\left(\Omega_{k}\right)+E\left\{G_{\text {leak }}+G_{\text {int }}+G_{V}\right\}
$$

where

$G_{\text {leak }}$ system leakage error with $E\left\{G_{\text {leak }}\right\}=O(((2 n+$ 1) $\left./ N)^{R+2}\right)=O\left(n^{R+2}\right)$. This error is correlated with the input and, hence, with $K_{n}$;

$G_{\text {int }}$ polynomial interpolation error with $E\left\{G_{\text {int }}\right\}=$ $\left(G_{k}^{(R+1)}\left(\Omega_{k}\right)\right) O\left(((2 n+1) / N)^{R+1}\right)=O\left(n^{R+1}\right)$. This error stems from the remainder of the polynomial interpolation of the FRF over $2 n+1$ frequencies. The errors $E\left\{G_{\text {leak }}\right\}$ and $E\left\{G_{\text {int }}\right\}$ clearly show that the bias error increases with the local bandwidth $n$ of the LPM;

$G_{V} \quad$ error due to the disturbing noise with $E\left\{G_{V}\right\}=0$.

The variance on the FRF estimate $\hat{G}_{k}\left(\Omega_{k}\right)$ is

$$
\hat{\sigma}_{\hat{G}}^{2}(k)=\sigma_{G_{V}}^{2}(k)+\sigma_{\text {leak } G}^{2}(k)+\sigma_{\operatorname{int} G}^{2}(k)
$$

where

$\sigma_{G_{V}}^{2}(k) \quad=O\left(n_{\theta} /(2 n+1)\right)=O(2(R+1) /(2 n+1)) ; \quad$ the variance due to the disturbing noise, with $n_{\theta}=$ $2(R+1)$ being the number of complex parameters to be estimated in polynomial approximation expressions using $2 n+1$ complex equations. The disturbing noise variance is directly proportional to the number of parameters to be estimated and inversely proportional to the local bandwidth used. Thus, the noise variance is growing proportional 


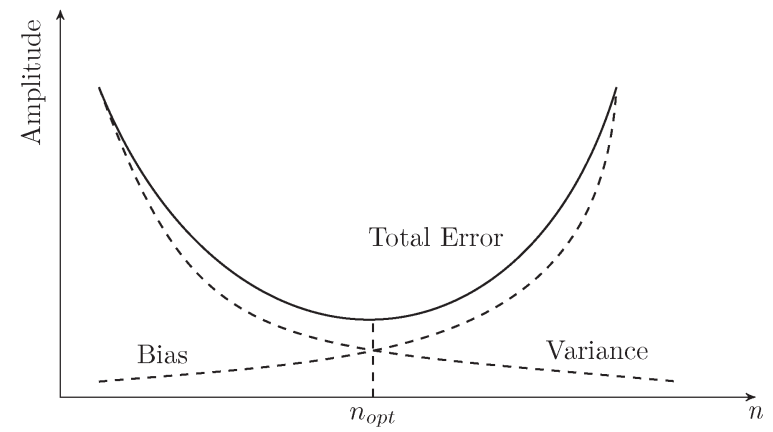

Fig. 1. Illustration of the behavior of the bias error and variance error with respect to the local bandwidth $(n)$.

with the number of parameters. If a large local bandwidth is used, the variance due to the disturbing noise will be reduced;

$\sigma_{\text {leakG }}^{2}(k)=O\left((n / N)^{(2 R+3)}\right)$; the variance of the leakage error, and it grows with $n$;

$\sigma_{\text {intG }}^{2}(k)=\left(\left|G_{k}^{(R+1)}\left(\Omega_{k}\right)\right|^{2}\right) O\left((n / N)^{2(R+1)}\right)$; the variance of the interpolation error, and it also grows with $n$.

By selecting a larger value of $n$, the noise sensitivity of $\hat{G}_{k}\left(\Omega_{k}\right)$ will be reduced (assuming that $\sigma_{G_{V}}^{2}$ dominates) at a cost of a growing interpolation error. The local bandwidth should be set to the smallest value (the minimal local bandwidth), in the absence of disturbing noise.

From Fig. 1, we can clearly understand that the bias error increases with the local bandwidth $(n)$, and variance error due to the disturbing noise decreases with it. We need to make the bias-variance tradeoff so that the improved local bandwidth $\left(n_{\mathrm{opt}}\right)$ at the local minimum of the total error can be selected.

\section{B. Improved Balance of Model Errors-Noise (Bias-Variance)}

1) Choice of the Polynomial Order R: Assume a fixed local bandwidth $n$. The noise interpolation error is $E\left\{G_{\text {int }}\right\}=$ $O\left(((2 n+1) / N)^{R+1}\right)$, and the ratio $((2 n+1) / N) \ll 1$; hence, $E\left\{G_{\text {int }}\right\}$ decreases with $R$. Thus, increasing $R$ will reduce the bias errors, but the calculation time increases proportionally as an $O\left(R^{3}\right)$ due to the larger number of parameters $n_{\theta}=2(R+1)$ to be estimated. In this paper, we put $R=2$. Optimizing the choice of $R$ is out of the scope of this paper.

2) Choice of the Local Bandwidth n: Assume a fixed polynomial order $R=2$. The bias error $E\left\{G_{\text {leak }}+G_{\text {int }}\right\}=$ $O\left(n^{R+1}\right)+O\left(n^{R+2}\right)$ increases with the local bandwidth $n$, and the noise variance $\sigma_{G_{V}}^{2}(k)=O(2(R+1) /(2 n+1))$ decreases with it. At each frequency, the improved value of $n$ is selected. In Section IV, we discuss the choice of improved local bandwidth with the bias-variance tradeoff.

\section{SElection of the Improved Local Bandwidth}

To get a lower noise sensitivity for a fixed polynomial order $R$, the local bandwidth $n$ of the LPM has to be tuned. If $n$ is the local bandwidth of the LPM, then $2 n+1$ neighboring frequency lines combine to generate the local point estimate (the FRF $\hat{G}_{k}\left(\Omega_{k}\right)$, the estimate of $\hat{G}$ at frequency $\Omega_{k}$, obtained from a local bandwidth centered around frequency $k$ ). In Section IV-A, we describe the basic idea of the improved

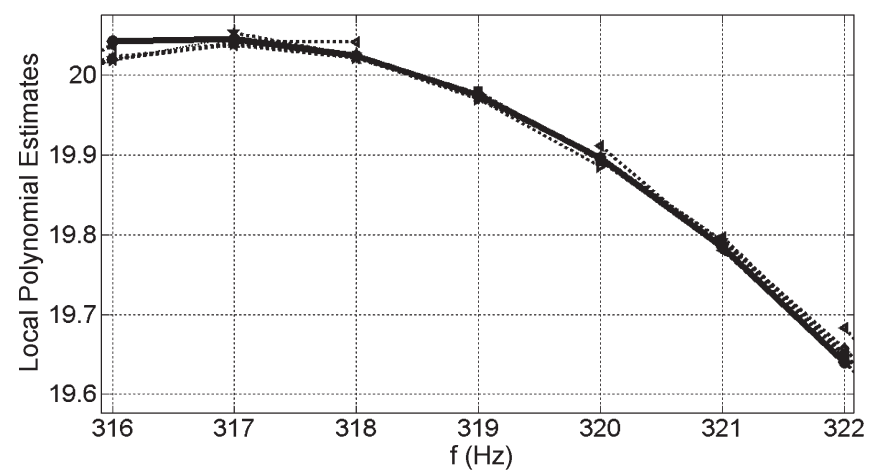

Fig. 2. Multiple local polynomial estimates for local bandwidth $n=3$ (without disturbing noise). (Bold line marked with •) FRF estimate $\hat{G}_{k}\left(\Omega_{k}\right)$. (Dotted lines) FRF estimates $\hat{G}_{k+r}\left(\Omega_{k+r}\right)$ with $r=[-3, \ldots, 0, \ldots, 3]$.

local bandwidth choice; next, we discuss how to estimate the FRF at the neighboring frequencies and how to compute the maximum deviation in Sections IV-B and IV-C, respectively. We discuss the generalized expressions for the FRFs at the neighboring frequencies and for the maximum deviation in Section IV-D. Next, we discuss the estimation of the improved FRF by selecting the improved local bandwidth in Section IV-E, followed by elimination of spikes in the improved local bandwidth choice in Section IV-F.

\section{A. Improved Local Bandwidth Choice: Basic Idea}

We have looked for number of principles for the selection of the improved local bandwidth; the first one is based on a classical Akaike information criterion (AIC criterion) [3], [19], [20]. The AIC criterion failed because, in [20], it was assumed that the variance of the errors does not vary within the local frequency interval $(k+[-n, \ldots, n])$, but often, the noise variance varies very fast in the frequency interval particularly if larger intervals are considered. Thus, we have looked for a more robust criterion which can also consider the fast variation of the noise variance. In this paper, we propose a new criterion for the improved local bandwidth choice, without any assumption and which works well at all frequencies.

The basic idea for the choice of the improved local bandwidth is as follows.

1) For a given local bandwidth $n$, select a frequency interval $k+r=k+[-n, \ldots, 0, \ldots, n]=[k-n, \ldots$, $0, \ldots, k+n]$ (of length $2 n+1$ ) around a frequency $\Omega_{k}$ of interest, with $r=[-n,-n+1, \ldots,-1,0,1, \ldots, n-$ $1, n]$ being the neighboring frequency points.

2) Fit a polynomial to input-output frequency data in the interval using a least squares approach (5) and (7).

3) Compute the maximum of the absolute value of the difference among the polynomial function/FRF estimated at $\Omega_{k}\left(\hat{G}_{k}\left(\Omega_{k}\right)\right)$, centered around frequency $k$, and the FRFs evaluated at the frequencies $\Omega_{k+r}\left(\hat{G}_{k+r}\left(\Omega_{k+r}\right)\right)$, centered around the neighboring frequencies $k+r$, within the same interval (16) and (17).

4) Repeat steps 1)-3) for a range of local bandwidths $n_{\text {All }}=$ $\left[n_{1}, n_{2}, \ldots, n_{q-1}, n_{q}\right]$.

5) Select the improved local bandwidth as the bandwidth that gives the minimum value in step 3) (20). 


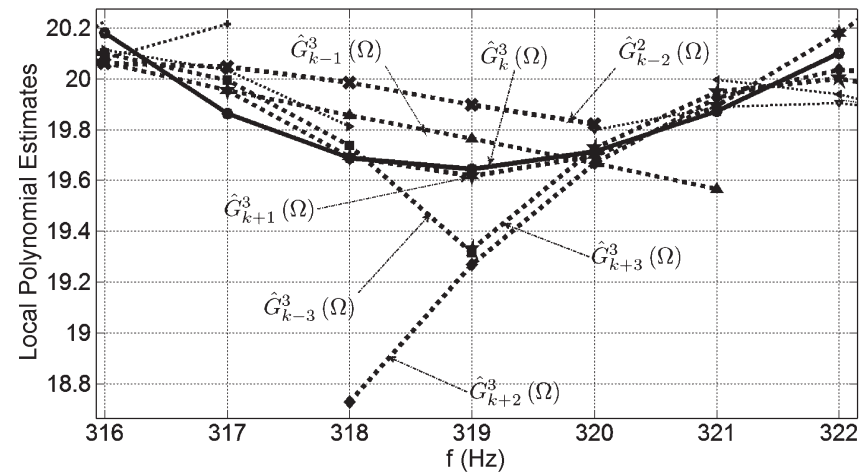

Fig. 3. Multiple local polynomial estimates for local bandwidth $n=3$ (with disturbing noise being added). (Bold line marked with •) FRF estimate $\hat{G}_{k}\left(\Omega_{k}\right)$. (Dotted lines) FRF estimates $\hat{G}_{k+r}\left(\Omega_{k+r}\right)$ with $r=$ $[-3, \ldots, 0, \ldots, 3]$.

We plot the local polynomial estimates in the interval $[k-$ $n, \ldots, 0, \ldots, k+n]$ using the polynomial function evaluated at $\Omega_{k}$ and at the neighboring frequencies. Ideally, without disturbing noise, the local polynomial estimates merge with each other (Fig. 2). If the disturbing noise is added to the output, the local polynomial estimates will not merge (Fig. 3); there is a deviation among the FRF estimated at $\Omega_{k}$ centered around the frequency $k$ and the FRFs estimated at the frequencies $\Omega_{k+r}$ centered around the frequencies $k+r$ within the same interval.

This paper considers the worst case scenario of the local polynomial estimates, i.e., estimate the maximum deviation among the FRF centered around frequency $k$ and the FRFs centered around frequencies $k+r$ in the interval $[k-$ $n, \ldots, 0, \ldots, k+n]$, for a range of local bandwidths $n_{\text {All }}$. This is the reason for selecting the cost function as the maximum of the absolute value of the deviation among the FRF $\hat{G}_{k}\left(\Omega_{k}\right)$ and the FRFs $\hat{G}_{k+r}\left(\Omega_{k+r}\right)$ in a given frequency interval. Finally, for the improved local bandwidth choice, consider the least worst case situation, i.e., minimize the cost function. We discuss the first two steps in the choice of the improved local bandwidth in Section IV-B.

\section{B. Estimation of the FRF at the Neighboring Frequencies in the LPM}

Estimate the FRF $\hat{G}_{k}\left(\Omega_{k}\right)$ at a frequency $\Omega_{k}$, centered around a frequency $k$, using a linear least squares procedure [by solving (7)]. Consider a frequency interval $k+r$ around the frequency $\Omega_{k}$. For a given local bandwidth $n$, the FRFs evaluated at the neighboring frequencies $\Omega_{k+r}$ [(5)] within the interval $k+r$, centered around frequencies $k+r$, are

$$
\hat{G}_{k+r}\left(\Omega_{k+r}\right)=\hat{G}_{k}\left(\Omega_{k}\right)+g_{1}\left(\Omega_{k}\right) r+g_{2}\left(\Omega_{k}\right) r^{2} .
$$

Similarly, the estimated FRFs at frequencies $\Omega_{k+r+i}$ are

$$
\begin{aligned}
\hat{G}_{k+r}\left(\Omega_{k+r+i}\right)=\hat{G}_{k}\left(\Omega_{k+i}\right)+g_{1} & \left(\Omega_{k+i}\right)(r+i) \\
& +g_{2}\left(\Omega_{k+i}\right)(r+i)^{2}
\end{aligned}
$$

with $r=[-n, \ldots,-1,0,1, \ldots, n]$ being the neighboring frequency points. We discuss step 3 ) in the improved local bandwidth choice in Section IV-C.

\section{Estimation of the Maximum of the Absolute Value of the Deviation}

The maximum of the absolute value of the deviation, among the estimated FRF $\hat{G}_{k}\left(\Omega_{k}\right)$, at frequency $\Omega_{k}$ centered around frequency $k$, and the FRFs evaluated $\hat{G}_{k+r}\left(\Omega_{k+r}\right)$, centered around frequencies $k+r$, is

$$
\delta \hat{G}_{\max }\left(\Omega_{k}\right)=\max \left(\operatorname{abs}\left(\delta G\left(\Omega_{k}\right)\right)\right)
$$

where $\delta G\left(\Omega_{k}\right)$ is the deviation among the FRF $\hat{G}_{k}\left(\Omega_{k}\right)$ and the FRFs $\hat{G}_{k+r}\left(\Omega_{k+r}\right)$, at frequency $\Omega_{k}$ which is given hereinafter

$$
\delta G\left(\Omega_{k}\right)=\left[\left(\hat{G}_{k}\left(\Omega_{k}\right)\right)-\left(\hat{G}_{k+r}\left(\Omega_{k+r}\right)\right)\right] .
$$

Similarly, the maximum deviation at a frequency $\Omega_{k+i}$ is

$$
\delta \hat{G}_{\max }\left(\Omega_{k+i}\right)=\max \left(\operatorname{abs}\left(\delta G\left(\Omega_{k+i}\right)\right)\right)
$$

where

$$
\delta G\left(\Omega_{k+i}\right)=\left[\left(\hat{G}_{k}\left(\Omega_{k+i}\right)\right)-\left(\hat{G}_{k+r}\left(\Omega_{k+i+r}\right)\right)\right] .
$$

Fig. 2 shows the multiple local polynomial estimates for a local bandwidth of $n=3$ without disturbing noise (all local polynomial estimates merge with each other). Fig. 3 shows the multiple local polynomial estimates with disturbing noise. From Fig. 3, we can clearly see that, at a frequency $\Omega_{319}$, $2 n+1=7$ neighboring FRF estimates combine to generate the FRF $\hat{G}_{319}\left(\Omega_{319}\right)$. We discuss steps 4$)$ and 5$)$ in the choice of the improved local bandwidth in detail, in Sections IV-D and IV-E, respectively.

\section{Generalized Expressions for a Given Local Bandwidth $n_{i}$}

To find the improved local bandwidth, we need to estimate the FRFs and the maximum deviations for a range of local bandwidths $n_{i}$ with $i=1,2, \ldots, q$. In this section, we generalize all expressions in Sections IV-B and IV-C [(14)-(18)], for a given local bandwidth $n_{i}$.

We denote the estimated FRF at a given frequency $\Omega_{k}$, obtained from a linear least squares procedure as $\hat{G}_{k}^{n_{i}}\left(\Omega_{k}\right)$. Following the same notation, the generalized expression for the FRFs evaluated at the neighboring frequencies $\Omega_{k+r}$ within the interval $k+r$, centered around the frequencies $k+r$, is

$$
\hat{G}_{k+r}^{n_{i}}\left(\Omega_{k+r}\right)=\hat{G}_{k}^{n_{i}}\left(\Omega_{k}\right)+g_{1}^{n_{i}}\left(\Omega_{k}\right) r+g_{2}^{n_{i}}\left(\Omega_{k}\right) r^{2}
$$

with $r=\left[-n_{i}, \ldots, 0, \ldots, n_{i}\right]$ being the neighboring frequency points. The coefficients $g_{1}^{n_{i}}$ and $g_{2}^{n_{i}}$ are estimated for each local bandwidth $\left(n_{i}\right)$ using a linear least squares procedure, as discussed in Section II.

The generalized expression for the maximum deviation at frequency $\Omega_{k}$ among the estimated FRF $\hat{G}\left(\Omega_{k}\right)$ and the FRFs evaluated at the center frequencies $k+r$ for a given bandwidth $n_{i}$ is

$$
\delta \hat{G}_{\max }^{n_{i}}\left(\Omega_{k}\right)=\max \left(\operatorname{abs}\left(\delta G^{n_{i}}\left(\Omega_{k}\right)\right)\right)
$$

where $\delta G^{n_{i}}\left(\Omega_{k}\right)$ is the deviation among the estimated FRF $\hat{G}_{k}^{n_{i}}\left(\Omega_{k}\right)$ and the FRFs evaluated at the frequencies centered 
around the frequencies $k+r$, for a given local bandwidth $n_{i}$, and is given hereinafter

$$
\delta G^{n_{i}}\left(\Omega_{k}\right)=\left(\left(\hat{G}_{k}^{n_{i}}\left(\Omega_{k}\right)\right)-\left(\hat{G}_{k+r}^{n_{i}}\left(\Omega_{k+r}\right)\right)\right)
$$

with $r=\left[-n_{i}, \ldots,-1,0,1, \ldots, n_{i}\right]$ being the neighboring frequency points for a given local bandwidth $n_{i}$.

\section{E. Improved Local Bandwidth Selection}

Once the maximum deviations for all local bandwidths $n_{i}$, with $i=1,2, \ldots, q$, are calculated from (20), then, the improved local bandwidth $n_{\mathrm{opt}}$ at a given frequency $\Omega_{k}$ is that local bandwidth which produces the lowest value of all the maximum deviations.

The improved local bandwidth $n_{\mathrm{opt}}$ is given by

$$
n_{\mathrm{opt}}\left(\Omega_{k}\right)=\arg \min _{n_{i}}\left(\delta \hat{G}_{\max }^{n_{i}}\left(\Omega_{k}\right)\right) .
$$

The improved FRF is estimated as

$$
\hat{G}_{\text {improved }}\left(\Omega_{k}\right)=\hat{G}_{k}^{n_{\mathrm{opt}}}\left(\Omega_{k}\right) .
$$

The minimal FRF (FRF calculated with the smallest local bandwidth, $n=3$ ) at each frequency $\Omega_{k}$ is estimated as

$$
\hat{G}_{\text {minimal }}\left(\Omega_{k}\right)=\hat{G}_{k}^{3}\left(\Omega_{k}\right) \text {. }
$$

\section{F. Elimination of Spikes in the Improved Local Bandwidth at Resonance}

At some frequencies, it was observed that the improved local bandwidth around a resonance frequency suddenly raised to the maximum local bandwidth used, leading to spikes in the local bandwidth selection. To eliminate this problem, the end edge frequencies are eliminated for all local bandwidths. It turned out from the simulations that this removed the problem of spikes.

\section{EXAMPLE}

\section{A. Introduction}

In this example, we show the improved local bandwidth selection on three simulations. To validate the proposed method, we solve the system with the disturbing noise added to the output as well as without disturbing noise. Simulation 1 discusses the effect of the disturbing noise on the improved local bandwidth selection, simulation 2 discusses the effect of the disturbing noise on the improved local bandwidth selection with more data points, and simulation 3 discusses the improved local bandwidth selection without disturbing noise. The degree $R$ of the polynomial interpolation is everywhere set equal to $R=2$.

\section{B. Simulation Setup}

The plant is a resonating fourth-order discrete-time system with $G_{0}\left(z^{-1}\right)=B\left(z^{-1}\right) / A\left(z^{-1}\right)$, with numerator coefficients $b=[0.1270,0.5080,0.7620,0.5180,0.1270]$ and denominator coefficients $a=[1.0000,-0.6072,1.3825,-0.6302,0.8869]$, generated with the Matlab instruction $[b, a]=\operatorname{cheby} 1(4,20$, $\left.6\left(f_{c} / f_{s}\right)\right)$ with $f_{c}=0.1 f_{s}, b=b \times 10$, and $b(4)=b(4)+0.01$.

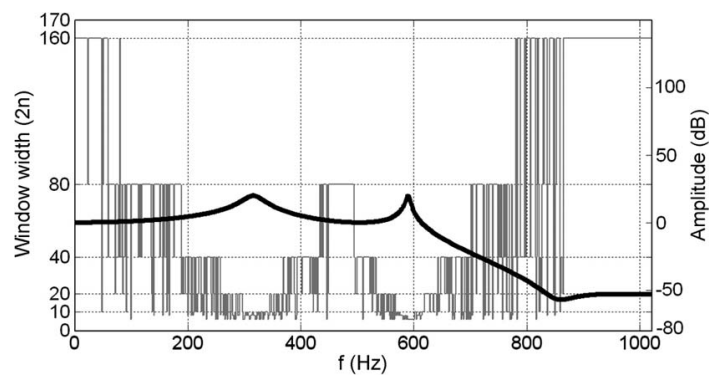

Fig. 4. Variation of the improved local bandwidth $\left(n_{\mathrm{opt}}\right)$ as a function of the frequency $\left(N_{T}=3072\right)$. (Bold black line) $G_{0}$. (Gray line) Improved window width $2 n_{\mathrm{opt}}$.

A filtered white noise sequence is used to excite the system; the coefficients of the generator filter are the following: $b_{\mathrm{Gen}}=[0.6389,1.2779,0.6389]$ and $a_{\mathrm{Gen}}=$ $[1.0000,1.1429,0.4128]$, generated with the Matlab instruction $\left[b_{\mathrm{Gen}}, a_{\mathrm{Gen}}\right]=\operatorname{butter}\left(2,2\left(f_{\mathrm{Gen}} / f_{s}\right)\right)$ with $f_{\mathrm{Gen}}=0.4 f_{s}$.

In simulations 1 and 2 , the output is disturbed by a white noise with signal-to-noise ratio at the output of $20 \mathrm{~dB}$ for all frequencies; for simulation 3 , no disturbing noise is added. The length of simulations 1 and 3 is $N_{T}=(1024+2048)$ points. The sampling frequency $f_{s}$ in all simulations is made equal to the number of data points excluding the transient data points, i.e., $f_{s}=N_{T}-N_{\text {Trans }}=$ 2048. The length of simulation 2 is $N_{T}=(1024+2048 \times 4)$ points. The first $N_{\text {Trans }}=1024$ points are used to eliminate the initial transient effects of all simulations.

The given system is solved with the window widths $w_{\text {All }}=$ $[6,8,10,20,40,80,160]$ or with the local bandwidths $n_{\mathrm{All}}=$ $[3,4,5,10,20,40,80]$. The term window width $w$ is twice of the local bandwidth, i.e., if $n$ is the local bandwidth, then the window width is $w=2 n$. All simulations (in Sections V and VI) are repeated for 1000 times.

\section{Simulation 1: With the Disturbing Noise Being Added to the Output and With a Simulation Length of $N_{T}=3072$}

The simulation results for the disturbing noise dominated case are shown in Figs. 4-6. The sampling frequency $f_{s}=2048 \mathrm{~Hz}$.

1) Results: In Fig. 4, we show the variation of the improved local bandwidth with respect to frequency. The local bandwidth drops to minimum value around the resonance frequency where the FRF varies fast and moves to larger values where the FRF varies smoothly. It should be emphasized that this automatic variation in the local bandwidth is obtained without any user interaction.

Fig. 5 shows the RMS error of the FRF estimates for improved as well as the minimal local bandwidths $\left(E_{\text {minimal }}\left(\Omega_{k}\right)=R M S\left(\hat{G}_{\text {minimal }}\left(\Omega_{k}\right)-G_{o}\left(\Omega_{k}\right)\right)\right)$. It clearly shows that the improved local bandwidth has much better noise sensitivity than the minimal local bandwidth. The zoomed view around the second resonance is also shown, which indicates that the RMS error for the improved local bandwidth ( $E_{\text {improved }}\left(\Omega_{k}\right)=R M S\left(\hat{G}_{\text {improved }}\left(\Omega_{k}\right)-\right.$ $\left.G_{o}\left(\Omega_{k}\right)\right)$ ) is slightly more than that for the minimal local bandwidth; the reason for this is a higher bias error for the improved local bandwidth which is clearly shown in Fig. 6. 

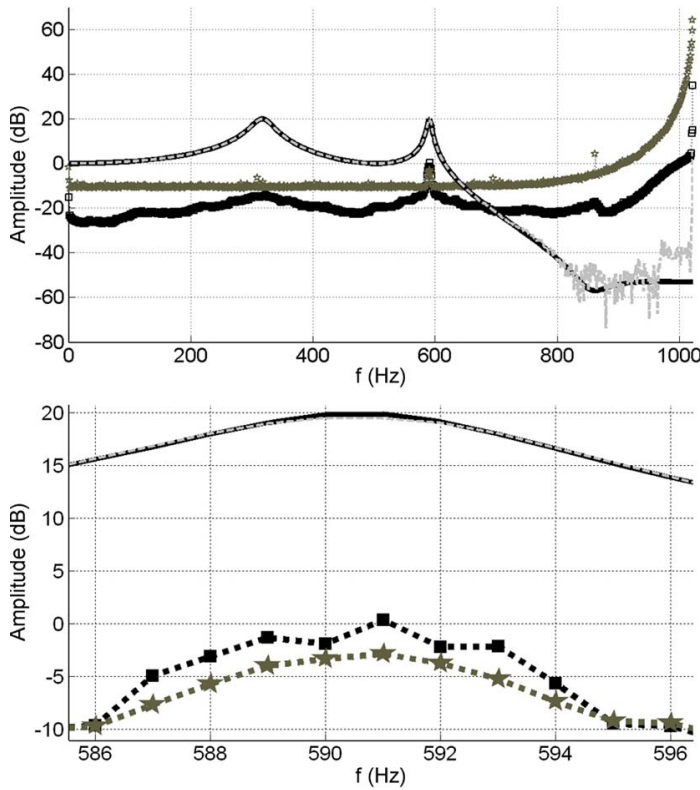

Fig. 5. Comparison of the RMS error of the FRF estimate $\left(N_{T}=3072\right)$. (Bottom) Zoom around the second resonance frequency. (Bold black line) $G_{0}$. (Dashed light gray line) Mean value of the estimated improved FRF. (Dark gray dotted line marked with $\star$ ) RMS error for the minimal local bandwidth. (Black dotted line marked with $\mathbf{}$ ) RMS error for the improved local bandwidth.
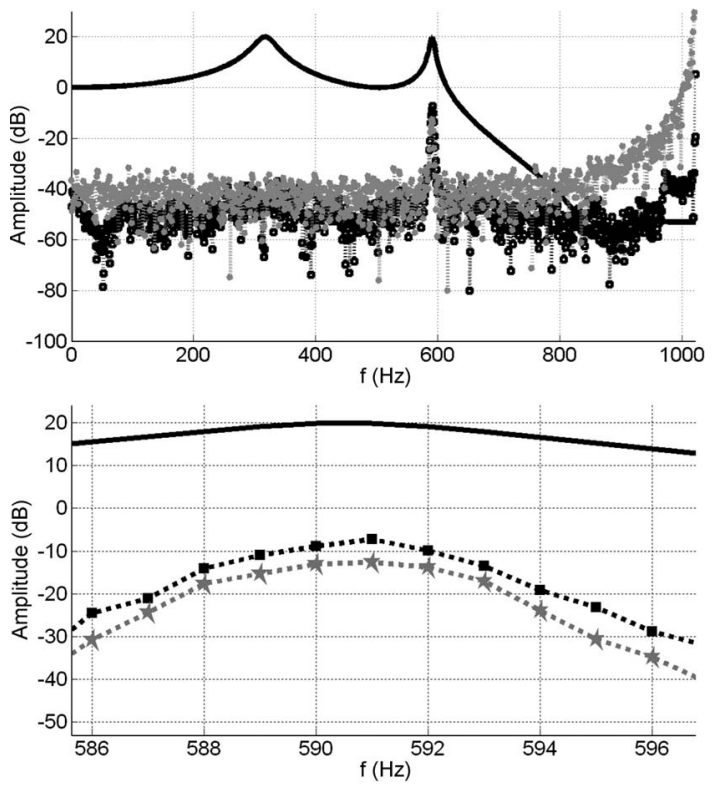

Fig. 6. Bias error on the estimated FRF. (Bottom) Zoom around the second resonance frequency $\left(N_{T}=3072\right)$. (Bold black line) $G_{0}$. (Dark gray dotted line marked with $\star$ ) Bias error on the estimated FRF for the minimal local bandwidth. (Black dotted line marked with $\mathbf{\square}$ ) Bias error on the estimated FRF for the improved local bandwidth.

2) Discussion: From these results, we can observe that the automatic selection of the local bandwidth results in the improved FRF. Where the FRF varies slowly, the method selects a wide frequency band to get a high noise rejection (assuming the bias error to be very small). Around the resonance frequency, the method selects the reduced local bandwidth, to keep the systematic errors under control (Fig. 4). The RMS error in the frequency band around the resonance frequency with the improved local bandwidth $\left(n_{\mathrm{opt}}\right)$ is approximately equal to that

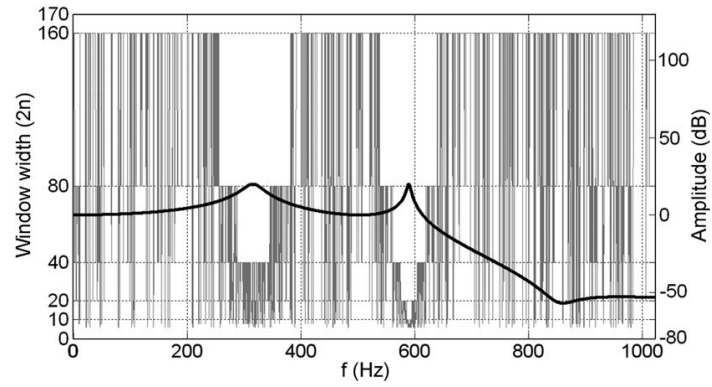

Fig. 7. Variation of the improved local bandwidth $\left(n_{\mathrm{opt}}\right)$ as a function of the frequency $\left(N_{T}=9216\right)$. (Bold black line) $G_{0}$. (Gray line) Improved window width $2 n_{\text {opt }}$.
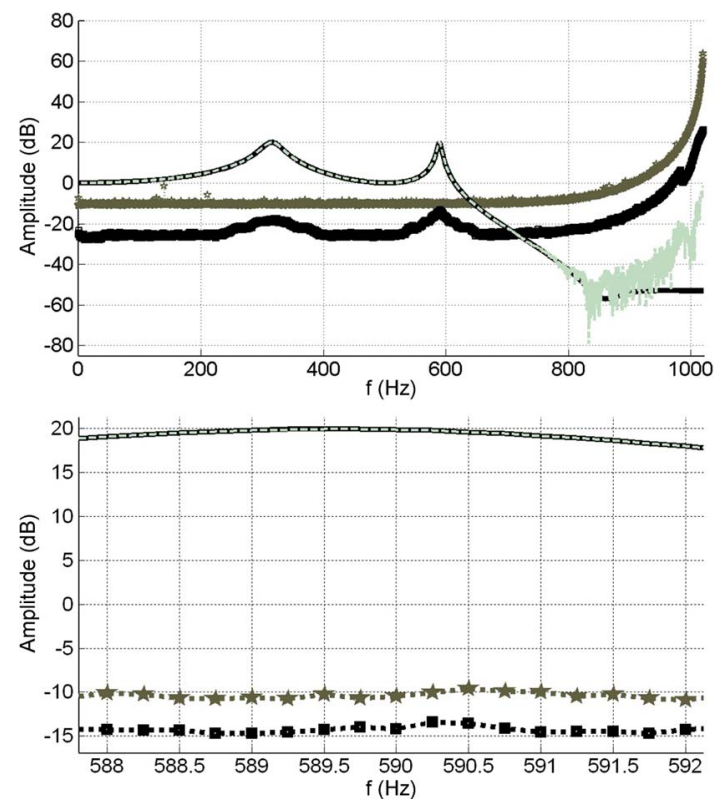

Fig. 8. Comparison of the RMS error of the FRF estimate $\left(N_{T}=9216\right)$. (Bottom) Zoom around second resonance frequency. (Bold black line) $G_{0}$. (Dashed light gray line) Mean value of the estimated improved FRF. (Dark gray dotted line marked with $\star$ ) RMS error for the minimal local bandwidth. (Black dotted line marked with $\mathbf{n}$ ) RMS error for the improved local bandwidth.

of the minimal local bandwidth $(n=3)$. Around the resonance frequency, the bias/leakage errors dominate (Fig. 6), so the errors are large. In the smooth frequency bands, where the noise error dominates, the RMS error of the improved local bandwidth is much better than that of the minimal local bandwidth (Fig. 5).

\section{Simulation 2: With the Disturbing Noise Being Added to the Output and With a Simulation Length of $N_{T}=9216$}

Simulation 2 results are shown in Figs. 7 and 8 with a simulation length of $N_{T}=N_{\text {Trans }}+N=1024+2048 \times 4=$ 9216 and with the sampling frequency $f_{s}=2048 \mathrm{~Hz}$.

1) Results: In Fig. 7, we show the variation of the improved local bandwidth with respect to frequency. In this case also, the local bandwidth drops to its minimum value around the resonance frequency where the FRF varies fast and moves to larger values where the FRF varies smoothly.

Fig. 8 shows the RMS error of the FRF estimates for the improved local bandwidth as well as the minimal local 


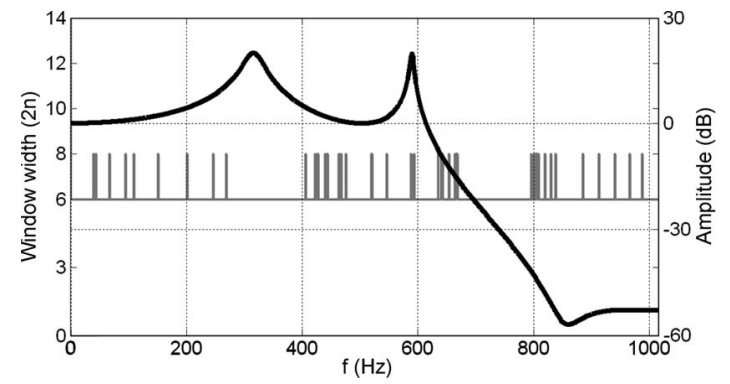

Fig. 9. Variation of the improved local bandwidth $\left(n_{\mathrm{opt}}\right)$ as a function of the frequency (without disturbing noise, $N_{T}=3072$ ). (Bold black line) $G_{0}$. (Gray line) Improved window width $2 n_{\text {opt }}$.
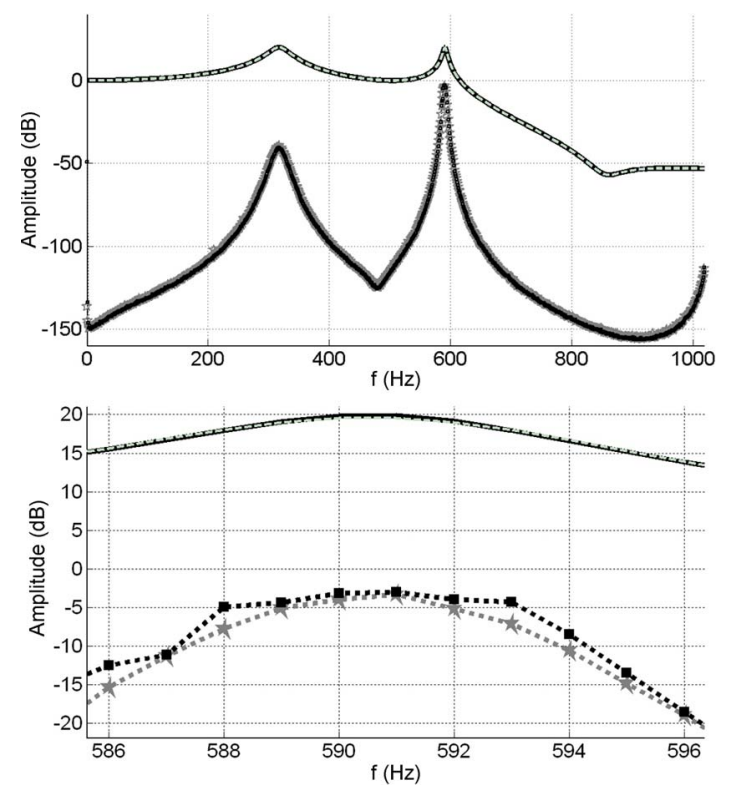

Fig. 10. Comparison of the RMS error of the FRF estimate (without disturbing noise, $N_{T}=3072$ ). (Bottom) Zoom around the second resonance frequency. (Bold black line) $G_{0}$. (Dashed light gray line) Mean value of the estimated improved FRF. (Dark gray dotted line marked with $\star$ ) RMS error for the minimal local bandwidth. (Black dotted line marked with $\mathbf{0})$ RMS error for the improved local bandwidth.

bandwidth. It clearly shows that the improved local bandwidth has much better noise sensitivity than the minimal local bandwidth in all frequency bands. The zoomed view around the second resonance shows that the RMS error is lower for the improved local bandwidth than that for the minimal local bandwidth.

2) Discussion: From the aforementioned simulation results, we can observe that, with more data points, the proposed method works very well. The RMS error on the estimated FRF for the improved local bandwidth is much better than that of the minimal local bandwidth in all frequency bands. At the sharp resonance, we got a slight improvement. Also, the number of frequencies in the $3-\mathrm{dB}$ bandwidth around the second resonance becomes very small (Fig. 8).

\section{E. Simulation 3: No Disturbing Noise Added to the Output and With a Simulation Length of $N_{T}=3072$}

The simulation results for the no-disturbing-noise case are shown in Figs. 9-11 with a simulation length of
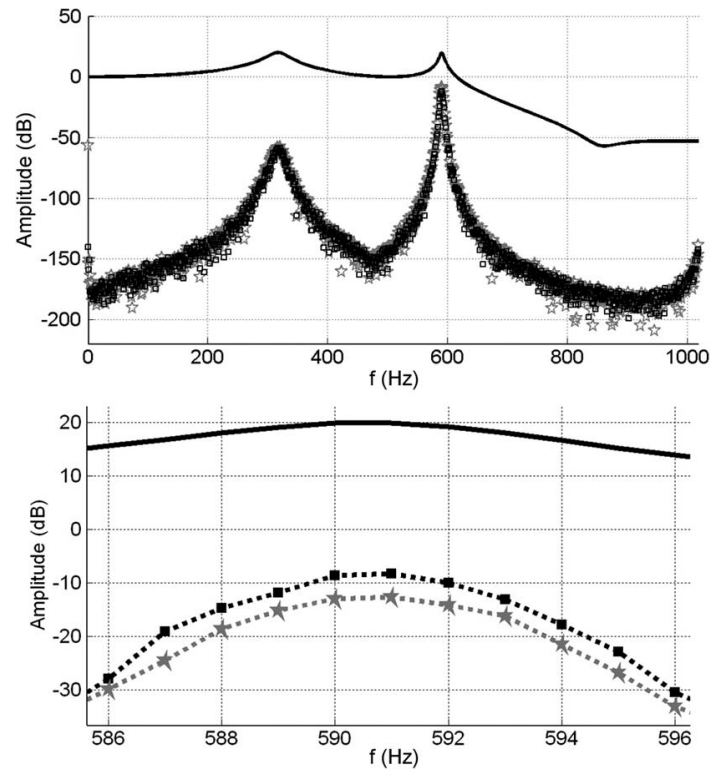

Fig. 11. Bias error on the estimated FRF (without disturbing noise, $N_{T}=$ 3072). (Bottom) Zoom around the second resonance frequency. (Bold black line) $G_{0}$. (Dark gray dotted line marked with $\star$ ) Bias error on the estimated FRF for the minimal local bandwidth. (Black dotted line marked with $\square$ ) Bias error on the estimated FRF for the improved local bandwidth.

$N_{T}=N_{\text {Trans }}+N=1024+2048=3072 . \quad$ The $\quad$ sampling frequency $f_{s}=2048 \mathrm{~Hz}$.

1) Results: Fig. 9 shows the variation of the improved local bandwidth $\left(n_{\mathrm{opt}}\right)$ with respect to the frequency. The improved local bandwidth automatically becomes minimum, without any user interaction, in almost all frequency bands. Fig. 10 shows the RMS error of the FRF estimates for the improved as well as the minimal local bandwidths. It shows that the improved and the minimal local bandwidths have almost the same RMS errors. Bottom one shows the zoomed view around the second resonance which indicates that the RMS error for the improved local bandwidth is slightly more than that of the minimal local bandwidth. Fig. 11 shows the bias error on the estimated FRF for the improved as well as the minimal local bandwidths.

2) Discussion: The proposed method works well for the no-disturbing-noise case. In almost all frequency bands, the improved local bandwidth drops to the minimum value except in very few frequencies (Fig. 9). Figs. 10 and 11 show that the LPM significantly reduces the leakage errors.

\section{F. Discussion}

A comparison of the improved local bandwidth (proposed method) and the minimal local bandwidth, with and without disturbing noise, is given hereinafter.

1) With Disturbing Noise: In the frequency bands around a resonance, the bias error dominates so that the local bandwidth $n$ automatically becomes smaller (Figs. 4 and 7), and in the remaining frequency bands, where the bias errors are smaller, reduced noise sensitivity is obtained by selecting the larger local bandwidth. This automatic selection of the local bandwidth requires no user interaction. 

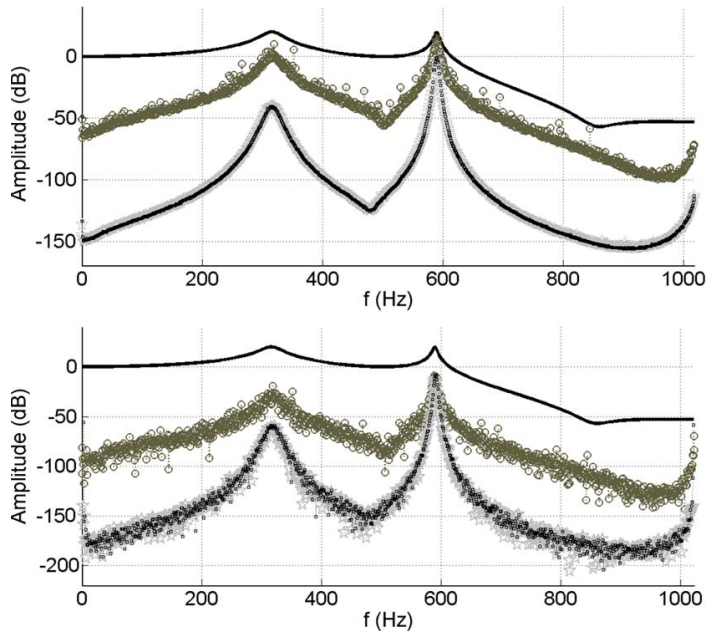

Fig. 12. (Top) RMS error on the estimated FRF (without disturbing noise, $M=8$ ). (Bottom) Bias error on the estimated FRF. (Bold black line) $G_{0}$. (Light gray dotted line marked with $\star$ ) Error (RMS/bias) on the estimated FRF for the minimal local bandwidth. (Black dotted line marked with $\square$ ) Error (RMS/bias) on the estimated FRF for the improved local bandwidth. (Dark gray dotted line marked with $o$ ) Error (RMS/bias) on the estimated FRF for the Hanning window.

a) With a Simulation Length of $N_{T}=3072$ : By comparing the RMS error of the estimated FRF for the improved and the minimal local bandwidths (Fig. 5), the improved local bandwidth is much better than the minimal local bandwidth except at some resonance frequencies, e.g., the bias error of the estimated FRF at the second resonance for the improved local bandwidth is higher than that of the minimal local bandwidth (Fig. 6).

b) With a Simulation Length of $N_{T}=9216$ : Comparison of the RMS error of the estimated FRF for the improved and the minimal local bandwidths shows that the improved local bandwidth is much better than the minimal local bandwidth in all frequency bands (Fig. 8). Moreover, we got an improvement at the sharp resonance.

2) Without Disturbing Noise and With a Simulation Length of $N_{T}=3072$ : The improved local bandwidth automatically becomes minimum at almost all frequencies (Fig. 9). Thus, the RMS error and the bias error of the estimated FRF for the improved local and the minimal local bandwidths are almost equal (Figs. 10 and 11).

\section{COMPARISON With LPM AND HANNING Window}

The proposed method (modified LPM) is compared with the conventional FRF estimation method like the Hanning window [1]. A theoretical analysis can also be found in [14]. The same system (described in Section V) is used for the comparison, with a simulation length of $N_{T}=1024+2048$ points, and the sampling frequency is $f_{s}=2048 \mathrm{~Hz}$. The full record length of $N=2048$ points is split into $M$ subblocks of length $N / M$ each for averaging purposes.

\section{A. Without Disturbing Noise}

In Fig. 12, we show the comparison of the RMS error and the bias error for the improved local bandwidth, the minimal
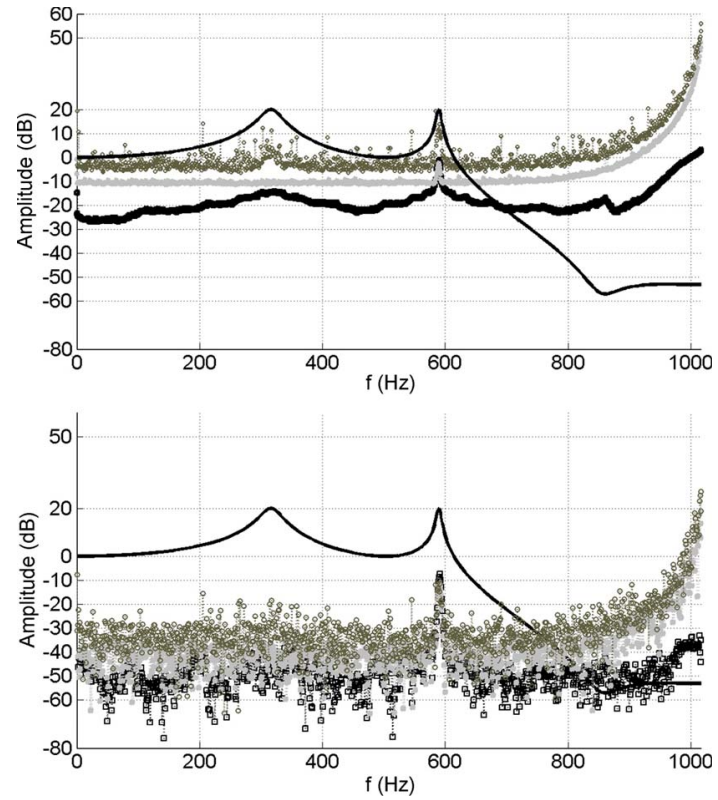

Fig. 13. (Top) RMS error on the estimated FRF (with disturbing noise, $M=1$ ). (Bottom) Bias error on the estimated FRF. (Bold black line) $G_{0}$. (Light gray dotted line marked with $\star$ ) Error (RMS/bias) on the estimated FRF for the minimal local bandwidth. (Black dotted line marked with $\mathbf{0})$ Error (RMS/bias) on the estimated FRF for the improved local bandwidth. (Dark gray dotted line marked with $o$ ) Error (RMS/bias) on the estimated FRF for the Hanning window.

local bandwidth, and the Hanning window for $M=8$. Since LPM significantly reduces the leakage errors, there is a huge difference between the RMS errors of the improved local bandwidth and the Hanning window.

\section{B. With Disturbing White Noise Added to the Output}

When there is noise on the output, the noise sensitivity of the LPM is more or less the same as that of the Hanning window (when no window width selection is done). The LPM works at full resolution $\left(f_{s} / N\right)$ while the Hanning window works at a reduced resolution $\left(M f_{s} / N\right)$ in order to average internally over a number of realizations $(M)$. Due to this averaging, the Hanning window seems to have a variance reduction of a factor $M$. In order to make a fair comparison for the noise sensitivity, this should be done at the same frequency resolution, and then, the Hanning window needs $M$ times more data.

For comparison of all the methods with the same frequency resolution, we made $M=1$. However, we are emphasizing in this paper that it is not a fair comparison for that reason. We can say that the equivalent gain is about $\operatorname{sqrt}(8)=9.03 \mathrm{~dB}$, which should be compensated for when interpreting these results. Around the resonance frequencies, the leakage error is still the dominant one, and the LPM wins again.

In Fig. 13, we show the comparison of the RMS error and the bias error with white noise for the improved local bandwidth, the minimal local bandwidth, and the Hanning window (for the same frequency resolution, i.e., $M=1$ ). Fig. 14 shows the comparison of the RMS error for all methods with disturbing white noise for $M=2,3$, and 4 . 


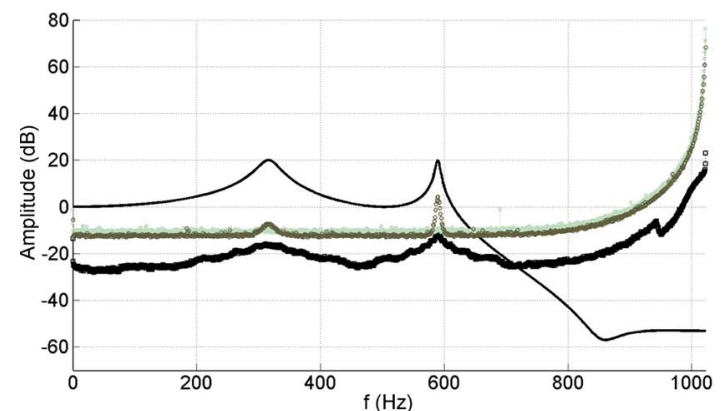

(a)

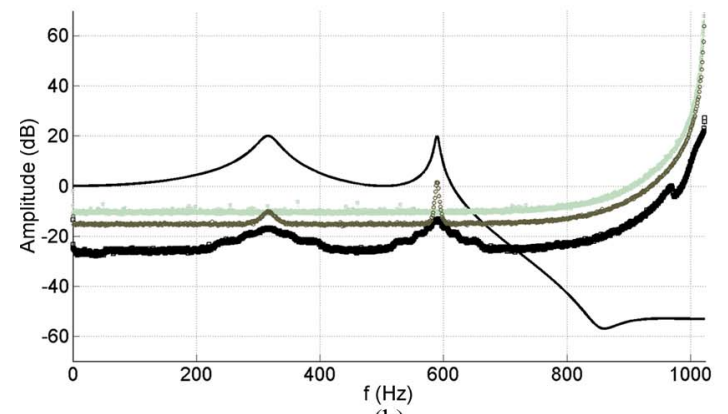

(b)

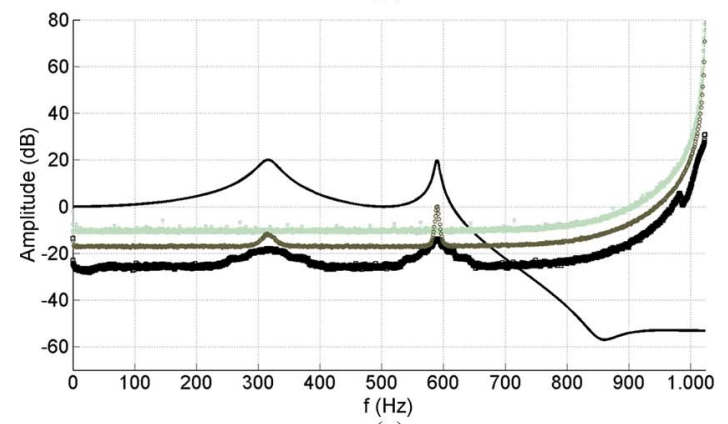

(c)

Fig. 14. RMS error on the estimated FRF (with disturbing white noise). (Bold black line) $G_{0}$. (Light gray dotted line marked with $\star$ ) RMS error on the estimated FRF for minimal local bandwidth. (Black dotted line marked with D) RMS error on the estimated FRF for improved local bandwidth. (Dark gray dotted line marked with $o$ ) RMS error on the estimated FRF for the Hanning window. (a) $M=2$. (b) $M=3$. (c) $M=4$.

\section{With Disturbing Colored Noise Added to the Output}

We generate the colored noise by passing the white noise through a filter generated with the Matlab instruction $\left[b_{\text {Noise }}, a_{\text {Noise }}\right]=$ cheby $1(5,10,8(f c / f s))$ with $f_{c} / f_{s}=0.1$. The coefficients of the filter are $b_{\text {Noise }}=$ $[0.1060,0.5301,1.0601,1.0601,0.5301,0.1060]$ and $a_{\text {Noise }}=$ $[1.0000,1.8963,1.6917,0.0613,-0.7006,-0.5562]$.

We compare all the methods for $M=1$ and 4. In Fig. 15, we show the comparison of the RMS error and the variation of improved local bandwidth with colored noise for the improved local bandwidth, the minimal local bandwidth, and the Hanning window.

\section{Discussion}

The proposed method is compared with classical windowing technique (Hanning window) in Figs. 12-15. Without disturbing noise, there is an amazing difference between the RMS and bias errors of the proposed method and the Hanning method.

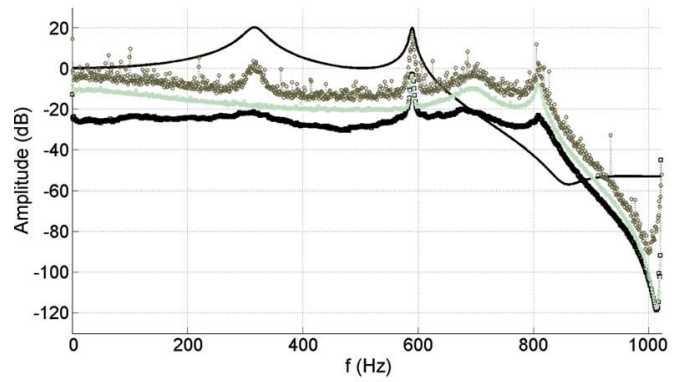

(a)

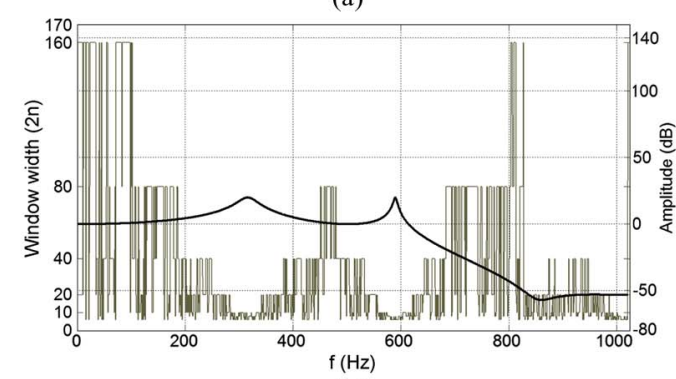

(b)

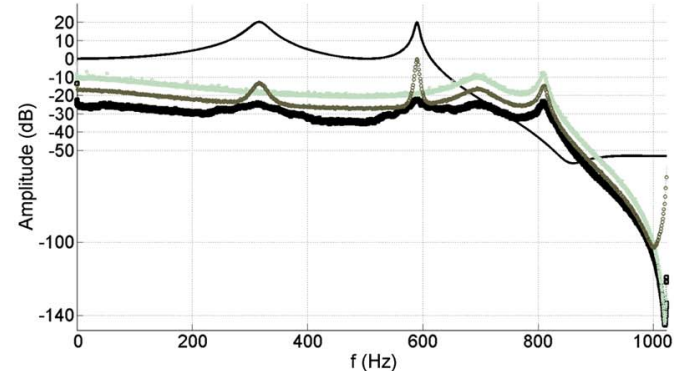

(c)

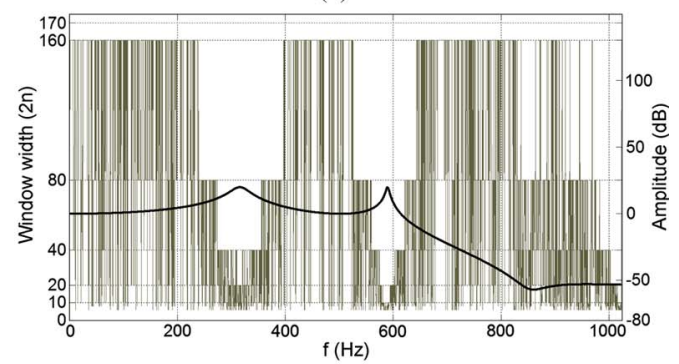

(d)

Fig. 15. (Bold black line) $G_{0}$. (Light gray dotted line marked with $\star$ ) RMS error on the estimated FRF for the minimal local bandwidth. (Black dotted line marked with $\square$ ) RMS error on the estimated FRF for the improved local bandwidth. (Dark gray dotted line marked with $o$ ) RMS error on the estimated FRF for the Hanning window. (Gray line in the improved local bandwidth variation) Improved window width $2 n_{\mathrm{opt}}$. (a) RMS error on the estimated FRF (with colored noise) for $M=1$. (b) Variation of the improved local bandwidth $\left(n_{\mathrm{opt}}\right)$ as a function of the frequency with colored noise for $M=1$. (c) RMS error on the estimated FRF (with colored noise) for $M=4$. (d) Variation of the improved local bandwidth $\left(n_{\mathrm{opt}}\right)$ as a function of the frequency with colored noise for $M=4$.

With the disturbing white noise being added to the output, the proposed method has superior noise sensitivity than the Hanning window in all frequency bands with the same frequency resolution, i.e., for $M=1$ (Fig. 13). The comparison of the RMS errors with $M=2,3$, and 4 shows that, even though there is loss of frequency resolution, the proposed method (improved LPM) works very well compared to the Hanning window and LPM (Fig. 14). The noise sensitivity of the improved LPM, for colored noise, is much better than that of the LPM and the Hanning window (Fig. 15). 


\section{CONCLUSION}

In this paper, we have used the LPM to get an improved FRF estimate using random excitations. Increased frequency resolution and high leakage rejection are the figures of merit of the LPM. High leakage reduction results in smaller bias and variance. In this paper, the algorithm is further extended to get much lower noise sensitivity by increasing the smoothening of the original LPM. An improved bias-variance tradeoff has been made. The proposed method works best when the disturbing noise dominates the leakage error.

The new method should only be used to produce smoother plots. If the data are further processed to fit a parametric model, the local bandwidth should always be selected to its minimum value in order to keep the bias errors as small as possible. The noise reduction is then made in the parametric step.

The final conclusion is that the improved local bandwidth selection yields very good noise sensitivity and very high quality nonparametric estimates of the FRF by automatic tuning of the parameter of the LPM without any user interaction.

\section{ACKNOWLEDGMENT}

The authors would like to thank the reviewers for their valuable suggestions.

\section{REFERENCES}

[1] J. S. Bendat and G. A. Piersol, Engineering Applications of Correlations and Spectral Analysis. New York: Wiley, 1980.

[2] D. R. Brillinger, Time Series: Data Analysis and Theory. New York: McGraw-Hill, 1981.

[3] L. Ljung, System Identification: Theory for the User (Second Edition). Upper Saddle River, NJ: Prentice-Hall, 1999.

[4] R. Pintelon and J. Schoukens, System Identification: A Frequency Domain Approach. Piscataway, NJ: IEEE Press, 2001.

[5] K. R. Godfrey, "Correlation methods," Automatica, vol. 16, no. 5, pp. 527-534, Sep. 1980.

[6] K. R. Godfrey, "The theory of the correlation methods of dynamic analysis and its application to industrial processes and nuclear power plant," Meas. Control, vol. 2, pp. T65-T72, May 1969.

[7] J. Schoukens, Y. Rolain, T. Dobrowiecki, and R. Pintelon, "Identification of linear systems with nonlinear distortions," Automatica, vol. 41, pp. 451-504, 2005.

[8] P. E. Wellstead, "Non-parametric methods of system identification," Automatica, vol. 17, no. 1, pp. 55-69, Jan. 1981.

[9] L. Rabiner and J. Allen, "On the implementation of a short-time spectral analysis method for system identification," IEEE Trans. Acoust., Speech, Signal Process., vol. ASSP-28, no. 1, pp. 69-78, Feb. 1980.

[10] J. L. Douce and L. Balmer, "Transient effects in spectrum estimation," Proc. Inst. Elect. Eng.D—Control Theory Appl., vol. 132, no. 1, pp. 2529, Jan. 1985.

[11] J. L. Douce and L. Balmer, "Statistics of frequency-response estimates," Proc. Inst. Elect. Eng.D-Control Theory Appl., vol. 137, no. 5, pp. 290296, Sep. 1990.

[12] J. Schoukens, Y. Rolain, and R. Pintelon, "Improved frequency response function measurements for random noise excitations," IEEE Trans. Instrum. Meas., vol. 47, no. 1, pp. 322-326, Feb. 1998.

[13] W. D. Widanage, J. L. Douce, and K. R. Godfrey, "Effects of overlapping and windowing on frequency response function estimates of systems with random inputs," IEEE Trans. Instrum. Meas., vol. 58, no. 1, pp. 214-220, Jan. 2009.

[14] R. Pintelon, J. Schoukens, and G. Vandersteen, "Estimation of nonparametric noise and FRF models for multivariable systems-Part I: Theory," Mech. Syst. Signal Process., vol. 24, no. 3, pp. 573-595, Apr. 2010.
[15] R. Pintelon, J. Schoukens, and G. Vandersteen, "Estimation of nonparametric noise and FRF models for multivariable systems-Part II: Applications and extensions," Mech. Syst. Signal Process., vol. 24, no. 3, pp. 596616, Apr. 2010.

[16] J. Schoukens, G. Vandersteen, K. Barbe, and R. Pintelon, "Nonparametric Preprocessing in System Identification: A Powerful Tool Theory," Eur. J. Control, vol. 15, no. 3/4, pp. 260-274, 2009.

[17] J. Schoukens, R. Pintelon, and H. Van hamme, "The interpolated fast Fourier transform: A comparative study," IEEE Trans. Instrum. Meas., vol. 41, no. 2, pp. 226-232, Apr. 1992.

[18] J. Antoni and J. Schoukens, "A comprehensive study of the bias and variance of frequency-response-function measurements: Improved window selection and overlapping strategies," Automatica, vol. 43, no. 10, pp. 1723-1736, Oct. 2007.

[19] T. Söderström and P. Stotia, System Identification. Englewood Cliffs, NJ: Prentice-Hall, 1989.

[20] J. Schoukens and R. Pintelon, "High quality frequency response function measurements without user interaction," in Proc. UKACC Int. Conf. CONTROL, Coventry, U.K., 2010, pp. 932-936.

[21] R. Pintelon, J. Schoukens, and G. Vandersteen, "Frequency domain system identification using arbitrary signals," IEEE Trans. Autom. Control, vol. 42, no. 12, pp. 1717-1720, Dec. 1997.

[22] T. McKelvey, "Frequency domain identification methods," Circuits Systems Signal Processing, vol. 21, pp. 39-55, 2002.

[23] J. C. Agüero, J. I. Yuz, G. C. Goodwin, and R. A. Delgado, "On the equivalence of time and frequency domain maximum likelihood estimation," Automatica, vol. 46, no. 2, pp. 260-270, Feb. 2010.

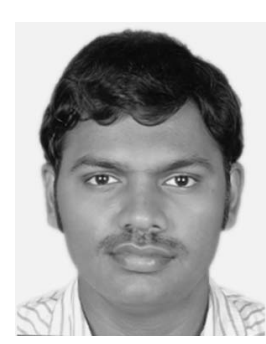

Prasanth Thummala (S'12) received the B.Tech. degree in electrical and electronics engineering from Acharya Nagarjuna University, Guntur, India, in 2008 and the M.Tech degree in control systems engineering, at the department of electrical engineering, from the Indian Institute of Technology Kharagpur, Kharagpur, India, in 2010. He is currently working toward the Ph.D. degree in the Power Electronics Group, Department of Electrical Engineering, Technical University of Denmark, Kongens Lyngby, Denmark.

From January 2011 to March 2011, he was a Visiting Researcher with the Department of Fundamental Electricity and Instrumentation (ELEC), Vrije Universiteit Brussel, Brussels, Belgium. His research interests include modeling and control of power converters, high-voltage switch-mode power converters for capacitive loads, and system identification.

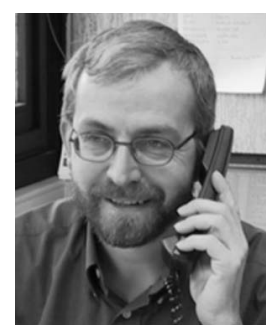

Johan Schoukens (F'97) received the degree of master in electrical engineering and the degree of doctor in engineering (Ph.D.) from the Vrije Universiteit Brussel (VUB), Brussels, Belgium, in 1980 and in 1985, respectively, and the Doctor Honoris Causa degree from the Budapest University of Technology and Economics, Budapest, Hungary, in 2011.

From 1981 to 2000 , he was a Researcher with the Belgian National Fund for Scientific Research (FWO-Vlaanderen) at the Department of Fundamental Electricity and Instrumentation (ELEC), VUB, where he is currently a full-time Professor in electrical engineering. His main research interests include system identification, signal processing, and measurement techniques.

Dr. Schoukens was the recipient of the 2002 Andrew R. Chi Best Paper Award of the IEEE Transactions on Instrumentation and MeaSUREMENT, the 2002 Society Distinguished Service Award from the IEEE Instrumentation and Measurement Society, and the 2007 Belgian Francqui Chair at the Université Libre de Bruxelles, Brussels, Belgium. Since 2010, he has been a member of Royal Flemish Academy of Belgium for Sciences and the Arts. 